\title{
Associação entre níveis de atividade física e transtorno mental comum em estudantes universitários
}

\author{
Association between levels of physical activity and common mental \\ disorder in university students
}

Adenice de Omena Silva, ${ }^{1}$ Jorge Lopes Cavalcante Neto ${ }^{2 *}$

ARTIGO ORIGINAL | ORIGINAL ARTICLE

Objetivou-se verificar se existe associação entre nível de atividade física (NAF) e transtorno menta comum (TMC) em universitários da área de saúde de uma universidade federal (UFAL). O estudo transversal com amostra de 220 universitários dos dois primeiros e dois últimos períodos de cada curso $(65.9 \%$ mulheres e $34.1 \%$ homens). Aplicou-se o Questionário Internacional de Atividade Física (IPAQ), o Self-Reporting Questionnaire (SRQ-20), e um questionário sociodemográfico. Utilizou-se o odds ratio (OR: IC95\%, $p<0.05$ ). Observou-se classificação positiva para TMC em $43.2 \%$ da amostra. Encontrou-se maior percentual de inativos com TMC (67.4\%) que ativos (32.6\%), com risco significativo $(\mathrm{OR}=3.202$, IC95\%: $1.830-5.600)$. Os estudantes dos primeiros períodos apresentaram-se mais ativos $(55.3 \%)$ em relação aos concluintes $(41.5 \%)$, com fator de proteção significativo $(\mathrm{OR}=0.575$, IC95\%: 0.337-0.980). Farmácia apresentou maior prevalência de TMC (24.2\%) e Nutrição a menor (9.5\%); por sua vez, Farmácia apresentou a maior prevalência de ativos (68.4\%), e Enfermagem de inativos $(58.3 \%)$. Observou-se que os estudantes inativos apresentaram três vezes mais chances de desenvolver TMC que os ativos, evidenciando que a graduação pode ser uma fase de risco para os universitários, seja devido aos diversos estressores, à ausência de hábitos saudáveis, e/ou à pouca e/ou inadequada prática de atividade física durante esse período.

Palavras-chave: atividade física, transtorno mental comum, saúde mental, estudantes universitários

ABSTRACT

This study aimed to investigate the association between physical activity level (PAL) and common mental disorders (CMD) in students of health programs at a federal university (UFAL). The crosssectional study comprised a sample of 220 students of the first two and last two periods of each program (65.9\% women; 34.1\% men). We used the International Physical Activity Questionnaire (IPAQ), the Self - Reporting Questionnaire (SRQ-20), and sociodemographic questionnaire. The odds ratios were calculated (OR: IC95\%, $p<0.05$ ). It was verified a positive CMD classification in $43.2 \%$ of the sample. A higher percentage of inactive students with TMC (67.4\%) than physically active students $(32.6 \%)$ was observed, with significant risk $(\mathrm{OR}=3.202$, IC95\%: $1.83-5.60)$. Undergraduate students in the first periods were more active $(55.3 \%)$ compared to counterparts $(41.5 \%)$, with a significant protective factor $(\mathrm{OR}=0.575$, IC95\%: 0.3370 .980$)$. Pharmacy had a higher prevalence of TMC $(24.20 \%)$ and Nutrition had the lowest $(9.50 \%)$. Pharmacy had a higher prevalence of physically active students $(68.40 \%)$, while Nursing had a higher prevalence of inactive students $(58.30 \%)$. It was observed that physically inactive students had three times more chance to develop TMC when compared with physically active students, showing that graduation can be a period of risk for the university, whether due to various stressors, lack of healthy habits, poor and/or inadequate practice of physical activity during this period.

Keywords: physical activity, common mental disorders, mental health, college students

Artigo recebido a 02.02.2013; Aceite 01.05.2013

${ }^{1}$ Universidade Federal de Alagoas, Alagoas, Brasil

${ }^{2}$ Universidade do Estado da Bahia - UNEB, Jacobina - BA, Brasil

* Autor correspondente: Universidade do Estado da Bahia, Departamento de Ciências Humanas, Campus IV, Avenida J.J. Seabra, 158, Estação, CEP: 44700-000 Jacobina - BA, Brasil; E-mail: jorgelcneto@hotmail.com 


\section{INTRODUÇÃO}

A prática regular de atividade física (AF) ajuda na promoção da saúde e na qualidade de vida, proporcionando benefícios significativos à saúde física e mental da população (Cid, Silva, \& Alves, 2007; Nahas \& Fonseca, 2004). O baixo nível de sua prática está associado ao desenvolvimento de doenças que afetam a saúde física e mental, resultando em maiores gastos para a saúde pública (Fontes \& Vianna, 2009).

A população tem acesso a informações sobre os prejuízos que o baixo nível de atividade física (NAF) causam à saúde, no entanto estudos recentes ainda mostram uma redução do NAF de diferentes populações, uma dessas populações é a de universitários (Fontes \& Vianna, 2009; Tondo, Silva, \& Roth, 2011). Um estudo realizado com 281 estudantes da área de saúde de Brasília, identificou alto índice de sedentarismo $(65.5 \%)$ (Marcodelli, Costa, \& Schmitz, 2008). Outro estudo utilizando o Questionário Internacional de Atividade Física (IPAQ-longo), realizado com discentes de uma universidade da Paraíba-Brasil, relatou a prevalência de baixo NAF entre os estudantes (31.2\%) (Fontes \& Vianna, 2009). Outra pesquisa utilizando o Questionário de Atividade Física Habitual (QAFH) para medir o NAF e as barreiras percebidas para a prática de $\mathrm{AF}$ entre estudantes de uma universidade federal do Rio Grande do Sul-Brasil, relatou a prevalência de pouca AF entre os estudantes (Tondo et al., 2011).

Os estudos citados mostram que a referente população tem reduzido a prática de $\mathrm{AF}$, o que poderá causar futuras perdas nas atividades académicas e na saúde em geral. Exigências da sociedade faz aumentar a concorrência entre os universitários antes mesmo de chegarem ao mercado profissional (Calais et al., 2007), podendo deixar os estudantes, mais ocupados e estressados com suas atividades, com as exigências do curso, com a aquisição de experiência e aprendizado.

$O$ ingresso na universidade pode expor os estudantes a estressores específicos (ansieda- de, problemas de moradia, distância da família) (Calais et al., 2007). E os últimos períodos na universidade podem sobrecarregá-los, devido à ansiedade da conclusão do curso, a dedicação e pressão psicológica durante a preparação do trabalho de conclusão de curso (TCC) (Calais et al., 2007), à incerteza do futuro profissional, que podem oferecer riscos à saúde mental (SM) dos estudantes podendo deixá-la debilitada (Polydoro, Primi, Serpa, Zaroni, \& Pombal, 2001).

A SM seria um equilíbrio mental entre sentimentos internos e experiências externas (Lino, 1997). Algumas doenças, agressões exógenas, excesso de trabalho, fadiga física e psíquica, e outros problemas devastam o equilíbrio mental, tornando-o angustiado, deprimido, nervoso e neurótico. Esses fatores ligados à predisposição genética são desencadeadores de transtornos mentais, que vão de um extremo psicótico (apresenta delírio) ou não-psicótico (não apresenta delírios). Logo, a reunião de alguns sintomas característicos de ansiedade, depressão e somatoformes compõem o que se pode denominar de transtornos mentais comuns (TMC) (Roeder, 1999), que são alterações do funcionamento da mente que podem prejudicar o indivíduo na vida familiar, social, pessoal, profissional, nos estudos, e na compreensão de si e dos outros (Ballone, 2008; Ludemir \& Filho, 2002).

O termo TMC é usado para indicar agrupamentos de sinais e sintomas associados a alterações de funcionamento da mente sem origem conhecida (Ludemir \& Filho, 2002), que são relacionados com problemas na SM. Para combater e/ou minimizar esses fatores de risco das afeções mentais, a AF é usada na prevenção e manutenção da SM, buscando desenvolver um estilo de vida ativo (Roeder, 1999).

A relação entre a SM e a prática de AF são processos particulares de expressão das condições e estilo de vida de uma população, logo, a prática de AF busca contribuir para a melhoria da SM das pessoas que possuem algum transtorno mental, melhorando a sua condição de vida, contribuindo para a melhoria do sono, do 
humor, da memória, da irritabilidade e na dificuldade de concentração (Araújo, Pinho, \& Almeida, 2005), que são fatores que caracterizam problemas de SM, e influenciam na saúde em geral. Universitários justificam que os hábitos estão mais sedentários após o ingresso na universidade, devido ao aumento nas horas de estudo. E que podem desenvolver algum tipo de TMC, devido à sobrecarga psicológica e a falta de AF (Fontes \& Vianna, 2009).

Na literatura, muitos estudos são referentes aos universitários (Silva, Silva, Silova, Souza, \& Tomazi, 2010; Souza, Santos, \& José, 2010), alguns avaliam o NAF, e outros buscam identificar problemas na SM dessa população (Calais et al., 2007; Fiorotti, Rossini, Borges, \& Miranda, 2010; Lima, Domingues, \& Cerqueira, 2006), mas, ainda é escasso o número de estudos que busquem associar esses fatores nesta população.

Assim, esse estudo procurou verificar a associação entre o NAF e a SM de estudantes universitários da área de saúde da Universidade Federal de Alagoas (UFAL), além de identificar e comparar os NAF, a presença de um provável TMC e os aspetos sociodemográficos entre os diferentes cursos.

\section{MÉTODO}

Trata-se de uma pesquisa de caráter quantitativo, de corte transversal. O estudo foi desenvolvido numa universidade federal (UFAL), em diferentes cursos ministrados num campus dessa instituição.

\section{Participantes}

A população alvo do estudo foi formada por estudantes dos cursos da área de saúde da UFAL (Educação Física, Odontologia, Farmácia, Nutrição, Medicina, Enfermagem e Ciências Biológicas), existindo cerca de 1930 estudantes de Bacharelado matriculados nos primeiros e últimos períodos.

A população de estudantes dos cursos da área de saúde da UFAL (Educação Física, Nutrição, Enfermagem, Odontologia, Medicina, Farmácia e Ciências Biológicas) era forma- da por 2523 universitários (de acordo com dados de matricula de 2011, coletados nas coordenações dos cursos), sendo 593 estudantes de Licenciatura e 1930 estudantes de Bacharelado. O presente estudo trabalhou apenas com a população de estudantes universitários de Bacharelado, por ser mais direcionado à área de saúde e ter um número significativo de pessoas.

O tamanho da amostra foi calculado a partir da estimativa de prevalência de transtornos mentais comuns em indivíduos jovens (Fiorotti et al., 2010). Tomou-se como base para o cálculo a presença de TMC como fator de risco. Dessa forma, tendo um risco alfa de $5 \%$ e um risco beta de $20 \%$, sendo necessários $10 \%$ do total de estudantes matriculados em cada curso, ou seja, Educação Física tinha 247 estudantes matriculados $(10 \%=25)$, Nutrição tinha 210 estudantes $(10 \%=21)$, Enfermagem tinha 237 estudantes $(10 \%=24)$, Odontologia tinha 305 estudantes $(10 \%=30)$, Farmácia tinha 265 estudantes $(10 \%=26)$ e Biologia tinha 186 estudantes $(10 \%=19)$.

O curso de Medicina terminou por não fazer parte da coleta de dados devido algumas restrições da coordenação do curso e do pequeno tempo disponível para cumpri-las. A percentagem da amostra de estudantes de medicina que seria coletada foi redistribuída com os outros cursos, com o intuito de se manter o número inicial da amostra.

Após a percentagem de estudantes de cada curso necessária para compor a amostra ser encontrada, ela foi dividida em $50 \%$ que cursem um dos períodos iniciais do curso $\left(1 \% / 2^{\circ}\right.$ período) e $50 \%$ que cursem um dos períodos finais $\left(7^{\circ} / 8^{\circ}\right.$ ou $\left.9^{\circ} / 10^{\circ}\right)$, A coleta nos períodos finais se deu dessa forma devido a alguns cursos que fazem parte do estudo, ou seja, Odontologia, Farmácia, Enfermagem e Nutrição terem como últimos períodos o $9^{\circ}$ e o $10^{\circ}$, enquanto os cursos de Educação Física e Biologia têm como períodos finais o $7^{\circ}$ e $8^{\circ}$.

Foi utilizado como critério de inclusão no estudo: alunos com idade $\geq 18$ anos, que estivessem cursando um dos dois primeiros perío- 
dos de um dos cursos de saúde ou um dos dois últimos períodos, devidamente matriculados num dos períodos citados no segundo semestre letivo de 2011.

$\mathrm{O}$ aluno já graduado, ou que estivesse participando de alguma intervenção do tipo psicológico ou psiquiátrico, foi excluído do estudo.

\section{Instrumentos e Procedimentos}

Para verificar o NAF foi utilizado o Questionário Internacional de Atividade Física (IPAQ), com a validade testada no Brasil (Matsudo et al., 2001), composto por 19 perguntas relacionadas às atividades realizadas uma semana antes de sua aplicação. A pontuação é obtida pela soma de dias, minutos ou horas das atividades, considerando a frequência, a intensidade e a duração.

Os dados foram tabulados, avaliados e classificados segundo o IPAQ (Matsudo et al., 2001; Silva et al., 2007), que divide e conceitua as categorias em: Sedentário; Insuficientemente Ativo, essa categoria divide-se em dois grupos, Insuficientemente Ativo A e Insuficientemente Ativo B; Ativo; Muito Ativo. Contudo para a facilitação das análises os grupos foram categorizados (Alves, Borges, Ribeiro, Gadelha, \& Santos, 2010; Guedes, Santos, \& Lopes, 2006; Rodrigues, Cheik, \& Mayer, 2008). Os universitários que foram classificados pelo IPAQ como muito ativo e ativo, foram categorizados como ativos, já os classificados como insuficientemente ativo $\mathrm{A} / \mathrm{B}$ e sedentários, passaram a ser classificados como Inativos.

O SRQ-20 é a versão de 20 itens do SRQ-30 para rastreamento de transtornos mentais nãopsicóticos (Mari \& Williams, 1986). As respostas são do tipo sim/não. Cada resposta afirmativa pontua com o valor 1 para compor o escore final por meio do somatório destes valores. $\mathrm{O}$ ponto de corte utilizado foi $\geq 7$ (Mari \& Wi lliams, 1986).

O questionário sociodemográfico utilizado foi elaborado tendo como base aspetos do domicílio e sociais, dicotomizados para análise. A idade foi dividida em 18-20 anos e 21-35 anos. A situação laboral foi dividida em traba- lha (quando possui atividade laboral remunerada) e não trabalha (quando não possui atividade laboral remunerada). O número de residentes no domicílio foi categorizado como apropriado (até 4 pessoas) ou inapropriado (mais de 4 pessoas). Quanto à situação socioeconómica, a amostra foi dividida inicialmente em quatro classes (A, B, C e D), dicotomizadas em seguida para a análise, em duas classes $(A+B)$ e $(C+D)$. Tal classificação resultou de uma pontuação construída segundo o poder económico das famílias e o grau de escolaridade do chefe da casa, através do que preconiza a Associação Brasileira de Empresas e Pesquisas (ABEP, 2003).

A pesquisa seguiu todas as prerrogativas da Declaração de Helsinque e da Resolução do Conselho Nacional de Saúde (CNS, 196/96) para pesquisas com seres humanos, sendo submetida e aprovada pelo Comité de Ética em Pesquisa da Universidade Federal de Alagoas (CEP/UFAL), recebendo a aprovação sob protocolo $n^{\circ}$ 013810/2011-38. O Termo de Consentimento Livre e Esclarecido (TCLE) foi entregue aos sujeitos da pesquisa para assinatura e consentimentos.

\section{Análise Estatística}

Para análise dos dados, foi utilizada a estatística descritiva, com a distribuição de frequência, média e desvio-padrão. Para a comparação dos grupos foi utilizado o teste quiquadrado. Para medida da associação foi utilizado o odds ratio (OR) com intervalo de confiança de $95 \%$ para verificar a associação entre os níveis de atividade física e o transtorno mental comum dos universitários investigados. O nível de significância utilizado foi $p<0.05$.

\section{RESULTADOS}

A amostra final foi composta por 220 estudantes universitários dos cursos da área de saúde da UFAL, Campus Maceió, sendo 65.9\% do sexo feminino e $34.1 \%$ do masculino. Já a média de idade do grupo foi de 22 anos. Enquanto $43.2 \%$ da amostra total apresentou classificação positiva para o TMC. 
A tabela 1 descreve as prevalências de TMC entre os cursos da área de saúde investigados no estudo. O curso de farmácia apresentou a maior prevalência (24.2\%). Já o curso de Nutrição apresentou a menor prevalência (9.5\%), dentre os seis cursos considerados.

Tabela 1

Prevalências de TMC entre os cursos da área de saúde $(N=220)$

\begin{tabular}{cc}
\hline Cursos & Prevalências de TMC \\
\hline Biologia & $16.8 \%$ \\
Educação Física & $12.6 \%$ \\
Enfermagem & $22.1 \%$ \\
Farmácia & $24.2 \%$ \\
Nutrição & $9.5 \%$ \\
Odontologia & $14.7 \%$ \\
\hline
\end{tabular}

A tabela 2 descreve as frequências de estudantes ativos e inativos entre os cursos da área de saúde investigados no estudo. O curso de Farmácia apresentou a maior prevalência de estudantes considerados ativos (68.4\%). Já o curso de Enfermagem apresentou a maior prevalência de estudantes considerados inativos (58.3\%).

Tabela 2

Frequências de estudantes ativos e inativos entre os cursos da área de saúde $(N=220)$

\begin{tabular}{ccc}
\hline Cursos & Ativos & Inativos \\
\hline Biologia & $53.1 \%$ & $46.9 \%$ \\
Educação Física & $65.8 \%$ & $34.2 \%$ \\
Enfermagem & $41.7 \%$ & $58.3 \%$ \\
Farmácia & $68.4 \%$ & $31.6 \%$ \\
Nutrição & $55.9 \%$ & $44.1 \%$ \\
Odontologia & $45.2 \%$ & $54.8 \%$ \\
\hline
\end{tabular}

A tabela 3 descreve os resultados dos possíveis fatores de risco para o TMC. Dentre os fatores considerados nenhum apresentou associação significativa. Vale ressaltar a tendência encontrada nas variáveis de sexo e escolaridade do chefe da casa.

A tabela 4 evidencia os fatores associados aos níveis de atividade física categorizados a partir do IPAQ. Observa-se um percentual maior de inativos com TMC $(67.4 \%)$ em relação aos ativos $(32.6 \%)$, com risco significativo $(\mathrm{OR}=3.202$, IC95\%: $1.83-5.60)$. Os estudantes com perfil académico inicial $\left(1^{\circ}\right.$ e $2^{\circ}$ períodos) apresentaram mais ativos (55.3\%) em relação aos alunos concluintes $\left(7^{\circ} / 8^{\circ} / 9^{\circ} / 10^{\circ}\right.$ períodos) com $41.5 \%$, indicando um fator de proteção significativo $(\mathrm{OR}=0.575$, IC95\%: 0.337-0.980). Cabe destacar a variável de situação laboral, que apresentou uma tendência, quando considerada na associação aos níveis de atividade física.

\section{DISCUSSÃO}

Apesar de existirem diversos estudos (Almeida et al., 2007; Amaral et al., 2008; Facundes \& Ludemir, 2005; Fontes \& Vianna, 2009; Guedes et al., 2006; Sakae, Padão, \& Jordana, 2010; Silva et al., 2007; Souza et al., 2010) referentes ao NAF e à SM de diferentes populações, ainda são escassas as pesquisas que verifiquem sua associação na população de universitários. Esses fatores terminam por dificultar a comparação dos resultados em estudos com tais objetivos.

Através de pesquisas utilizando o SRQ e outros questionários, que verificam a presença de TMC, alguns estudiosos (Baldisserotto, Filho, Nedel, \& Sakae, 2005; Cavestro \& Rocha, 2006) estimam que mais de $15 \%$ dos estudantes universitários apresentem ou possam vir a apresentar algum tipo de TMC durante a formação académica.

Já outros estudos (Lawlor \& Hopker, 2001; Sakae et al., 2010) têm demonstrado que a reunião de sintomas ansiosos, depressivos e somatoformes acometem diferentes populações, inclusive a de universitários, prejudicando a qualidade de vida. Entretanto, a prática de atividades físicas pode surtir efeito protetor aos sintomas do TMC (Faulkner \& Taylor, 2005), ou seja, se sua prática for regular poderá influenciar em uma melhor qualidade do sono, em melhorias nas funções cognitivas, no humor, na autoestima e no condicionamento físico, produzindo efeitos que protegem o 
Tabela 3

Fatores associados aos Transtornos Mentais Comuns (TMC) em estudantes universitários de cursos da área de saúde

\begin{tabular}{|c|c|c|c|c|}
\hline Variáveis & $\begin{array}{c}\text { Descrição da } \\
\text { amostra } \\
\text { n (\%) }\end{array}$ & $\begin{array}{c}\text { Prevalência de } \\
\text { TMC } \\
\text { n }(\%)\end{array}$ & OR (IC95\%) & $p$ \\
\hline \multicolumn{5}{|l|}{ Idade (anos) } \\
\hline $18-20$ & $81(36.8)$ & $37(45.7)$ & \multirow{2}{*}{$1.174(0.676-2.040)$} & \multirow{2}{*}{0.576} \\
\hline $21-35$ & $139(63.2)$ & $58(41.7)$ & & \\
\hline \multicolumn{5}{|l|}{ Sexo } \\
\hline Feminino & $145(65.9)$ & $69(47.6)$ & \multirow{2}{*}{$0.584(0.328-1.040)$} & \multirow{2}{*}{0.085} \\
\hline Masculino & $75(34.1)$ & $26(34.7)$ & & \\
\hline \multicolumn{5}{|l|}{ Classe social } \\
\hline$A+B$ & $110(50.2)$ & $43(39.1)$ & \multirow{2}{*}{$1.421(0.831-2.431)$} & \multirow{2}{*}{0.221} \\
\hline$C+D$ & $109(49.8)$ & $52(47.7)$ & & \\
\hline \multicolumn{5}{|l|}{ Perfil académico } \\
\hline Inicial & $114(51.8)$ & $49(43.0)$ & \multirow{2}{*}{$0.983(0.577-1.677)$} & \multirow{2}{*}{1.00} \\
\hline Final & $106(48.2)$ & $46(43.4)$ & & \\
\hline \multicolumn{5}{|l|}{ Trabalha atualmente } \\
\hline Sim & $62(28.3)$ & $22(35.5)$ & \multirow{2}{*}{$0.633(0.345-1.162)$} & \multirow{2}{*}{0.173} \\
\hline Não & $157(71.7)$ & $73(46.5)$ & & \\
\hline \multicolumn{5}{|l|}{ Número de pessoas no lar** } \\
\hline$\leq 4$ pessoas & $138(63.0)$ & 55 (39.9) & \multirow{2}{*}{$1.472(0.847-2.560)$} & \multirow{2}{*}{0.204} \\
\hline$>4$ pessoas & $81(37.0)$ & $40(49.4)$ & & \\
\hline \multicolumn{5}{|c|}{ Escolaridade do chefe da casa** } \\
\hline$\leq 5$ anos de estudo & $20(4.90)$ & $05(25.0)$ & \multirow{2}{*}{$0.393(0.138-1.125)$} & \multirow{2}{*}{0.097} \\
\hline$>5$ anos de estudo & $194(47.3)$ & $89(45.9)$ & & \\
\hline
\end{tabular}

Nota: * Nível de significância $p<0.05$ com a utilização do teste do Qui-quadrado; **Dados não analisados com a amostra total.

organismo contra um desequilíbrio na saúde mental e física, que poderiam levar um indivíduo a desenvolver um TMC (Calfas \& Taylor, 1994).

Os resultados encontrados quanto à associação do NAF e TMC mostram que dos estudantes ativos apenas $32.6 \%$ apresentavam predisposição a desenvolver algum tipo de TMC, já os inativos apresentaram $67.4 \%$ de predisposição a desenvolver algum tipo de TMC. Como não foi encontrado trabalho similar nas fontes consultadas, optou-se por descrever estudos de base populacional que contemplem em seus resultados essa associação. Num estudo de base populacional, realizado no Colorado, também foi encontrada associação entre a atividade física e a saúde mental da população estudada demonstrando que a população universitária se encontra em situação de risco (DemontHeinrich, 2009).

Foi encontrada maior prevalência de TMC em estudantes de Farmácia (24.2\%), seguido de Enfermagem com $22.1 \%$, Biologia com $16.8 \%$, Odontologia com $14.7 \%$, Educação Física com $12.6 \%$ e Nutrição com $9.5 \%$. Um estudo realizado na Universidade Federal de Pernambuco, com alunos dos cursos da área de saúde, sobre prevalência de TMC e utilizandose do SRQ-20 obteve como resultado uma taxa de TMC de $34.1 \%$ entre os participantes, sem, contudo, considerar os cursos separadamente, como no presente estudo (Facundes \& Ludemir, 2005).

$O$ presente estudo também mostra que quando verificada a frequência de estudantes ativos e inativos dos cursos da área da saúde, o 
Tabela 4

Fatores associados aos níveis de atividade física categorizados a partir do IPAQ em estudantes universitários de cursos da área de saúde

\begin{tabular}{|c|c|c|c|c|}
\hline Variáveis & $\begin{array}{l}\text { Ativo } \\
\mathrm{n}(\%)\end{array}$ & $\begin{array}{l}\text { Inativo } \\
\text { n (\%) }\end{array}$ & OR (IC95\%) & $p$ \\
\hline \multicolumn{5}{|l|}{$T M C$} \\
\hline Positivo & $31(32.6)$ & $64(67.4)$ & $3.202(1.830-5.600)$ & $0.001^{*}$ \\
\hline Negativo & $76(60.8)$ & $49(39.2)$ & & \\
\hline \multicolumn{5}{|l|}{ Idade (anos) } \\
\hline $18-20$ & $42(51.9)$ & $39(48.1)$ & $0.816(0.471-1.412)$ & 0.487 \\
\hline $21-35$ & $65(46.8)$ & $74(53.2)$ & & \\
\hline \multicolumn{5}{|l|}{ Sexo } \\
\hline Feminino & $67(46.2)$ & $78(53.8)$ & $0.752(0.430-1.314)$ & 0.324 \\
\hline Masculino & $40(53.3)$ & $35(46.7)$ & & \\
\hline \multicolumn{5}{|l|}{ Classe social } \\
\hline$A+B$ & $54(49.1)$ & $56(50.9)$ & $1.019(0.600-1.731)$ & 1.000 \\
\hline$C+D$ & $53(48.6)$ & $56(51.4)$ & & \\
\hline \multicolumn{5}{|l|}{ Perfil acadêmico } \\
\hline Inicial & $63(55.3)$ & $51(44.7)$ & $0.575(0.337-0.980)$ & $0.044^{*}$ \\
\hline Final & $44(41.5)$ & $62(58.5)$ & & \\
\hline \multicolumn{5}{|l|}{ Trabalha atualmente } \\
\hline $\operatorname{Sim}$ & $37(59.7)$ & $25(40.3)$ & $0.544(0.299-0.988)$ & 0.052 \\
\hline Não & $70(44.6)$ & $87(55.4)$ & & \\
\hline \multicolumn{5}{|l|}{ Número de pessoas no lar** } \\
\hline$\leq 4$ pessoas & $72(52.2)$ & $66(47.8)$ & $1.434(0.825-2.490)$ & 0.211 \\
\hline$>4$ pessoas & $35(43.2)$ & $46(56.8)$ & & \\
\hline \multicolumn{5}{|c|}{ Escolaridade do chefe da casa** } \\
\hline$\leq 5$ anos de estudo & $10(50.0)$ & $10(50.0)$ & $0.940(0.374-2.360)$ & 1.000 \\
\hline$>5$ anos de estudo & $94(48.5)$ & $100(51.5)$ & & \\
\hline
\end{tabular}

curso de Farmácia obteve os melhores resultados, sendo $68.4 \%$ estudantes ativos e $31.6 \%$ estudantes inativos. O curso de Educação Física teve como resultado $65.8 \%$ ativos e $34.2 \%$ inativos; Nutrição teve $55.9 \%$ ativos e $44.1 \%$ inativos; Biologia teve $53.1 \%$ ativos e $46.9 \%$ inativos; Odontologia teve $45.2 \%$ ativos e $54.8 \%$ inativos; Enfermagem teve $41.7 \%$ ativos e $58.3 \%$ inativos.

Os resultados encontrados no curso de Farmácia mostraram que os universitários apresentaram um maior nível de atividade física; no entanto, também apresentou a maior prevalência de TMC. Uma possível explicação para esses achados é que alguns desses estudantes podem estar realizando AF em excesso, em locais e horários inadequados, e isso pode estar contribuindo negativamente com a alta predisposição desses universitários ao desenvolvimento de TMC, visto que a atividade física é benéfica desde que seja realizada na frequência, na intensidade e na quantidade necessária ao bom funcionamento do organismo (Scully, Kremer, Meade, Grahan, \& Dudgeon, 1998), refletindo em pontos positivos para a boa saúde mental.

Dentre os resultados de prevalência de TMC o menor encontrado foi no curso de Nutrição, 
sendo que possível hipótese para o resultado encontrado é que os universitários do curso estão conseguindo um equilíbrio fundamental entre suas atividades do quotidiano, que inclui os estudos e outras tarefas diárias, e a prática adequada e regular de atividade física.

Os estudantes concluintes (2 últimos períodos) dos cursos apresentaram maior inatividade $(58.5 \%)$, já os iniciantes apresentaram $44.7 \%$, com associação significativa (OR= 0.575, IC95\%: 0.337-0.980). Resultados parecidos foram encontrados em outros estudos (Bray \& Born, 2004; Silva et al., 2007) respetivamente no Canadá e no Brasil, que identificaram a presença de estudantes mais ativos no início do curso que no término. Uma possível explicação seria que no início da vida académica os estudantes tentam equilibrar a vida de estudante com a vida pessoal e ao se aproximar do término do curso eles tendem a reduzir seu NAF devido à redução de horas e/ou dias disponíveis (devido a estágio, entrega de trabalho de conclusão de curso e ofertas de emprego), que pode influenciar inicialmente na frequência e na intensidade da prática de atividade física e posteriormente na ausência dela (Silva et al., 2007). Logo estar no período inicial é um fator de proteção para ser considerado ativo.

Neste estudo a prevalência de TMC foi maior no sexo feminino (47.6\%) comparativamente com o masculino (34.7\%), no entanto sem uma diferença estatisticamente significativa $(p=0.085)$. Resultados parecidos foram encontrados no estudo de Lima et al. (2006), realizado na cidade de Botucatu - São Paulo, onde verificaram a prevalência de TMC em estudantes de Medicina, obtendo como resultado uma prevalência de $47.6 \%$ entre as mulheres e $39.7 \%$ entre os homens. Outros estudos realizados em Pernambuco com cursos da área de saúde (Facundes \& Ludemir, 2005) e realizado em uma universidade da Bahia (Almeida et al., 2007) comprovam o resultado encontrado na população de universitários.

As características sociodemográficas foram apresentadas para caracterizar a amostra, além de terem sido utilizadas na realização das asso- ciações com o objetivo da pesquisa. Não houve associação estatisticamente significativa entre as condições sociodemográficas, o NAF e a SM dos universitários. A possível explicação é que devido o grupo de estudantes serem homogéneos em suas características sociodemográficas e económicas, as pequenas diferenças perderam sua importância; isso também aconteceu no estudo de Fiorotti et al. (2010) que verificavam TMC em estudantes de Medicina e no estudo de Lima et al. (2006) que verificaram a prevalência de fatores de risco para a saúde mental de estudantes de Medicina.

No entanto a variável de situação laboral apresentou uma tendência, quando considerada na associação aos níveis de atividade física, os estudantes que trabalham foram identificados como mais ativos (59.7\%), já os universitários que não trabalhavam (44.9\%) foram indicados como ativos. Isso pode ter acontecido devido à prática de atividade física realizada muitas vezes na própria função laboral que acaba por favorecer a prática regular de atividade física de universitários que trabalham. $\mathrm{O}$ resultado encontrado indicou uma tendência, talvez se o número da amostra fosse maior ou se abrangesse uma quantidade de indivíduos mais velhos o resultado encontrado pudesse ser significativo.

A categorização dos níveis de AF pode ter sido uma limitação dos instrumentos do estudo, talvez por não deixar explicito os dados encontrados com a aplicação do IPAQ e sua classificação antes da categorização, o que impede o conhecimento dos dados agrupados e da distribuição exata em cada uma delas (Guedes et al., 2006; Rodrigues et al., 2008). Deve ser considerado ainda que a equivalência das variáveis investigadas possa variar em função da percentagem da amostra (Hallal et al., 2005).

\section{CONCLUSÕES}

No presente estudo foi encontrada associação significativa entre os níveis de atividade física e saúde mental dos estudantes universitários dos cursos de saúde da UFAL, onde os 
estudantes classificados como inativos apresentaram três vezes mais possibilidades de desenvolver transtorno mental comum que os estudantes universitários ativos. Esse resultado contribui como evidência que o período de graduação pode ser uma fase de risco para os universitários, seja devido aos diversos estressores, à ausência de hábitos saudáveis, e à pouca e/ou inadequada prática de AF durante esse período.

Logo é de fundamental importância para a saúde dessa população que sejam elaboradas estratégias de prevenção e intervenção voltadas para esse grupo populacional, sobretudo considerando programas de atividade física como propostas para esse grupo na universidade.

Futuras pesquisas podem contribuir para uma melhor compreensão dos fatores de risco e seus efeitos no desenvolvimento de TMC e sua relação com o nível de atividade física em diferentes cursos universitários, visto que cada curso apresenta características próprias e, no entanto, compartilham de condições comuns que podem comprometer a saúde e prática de hábitos saudáveis dos estudantes durante a sua formação académica.

\section{Agradecimentos:}

Os autores agradecem às coordenações dos cursos de graduação da UFAL pelas informações e acesso dos pesquisadores no local.

\section{Conflito de Interesses:}

Nada a declarar.

Financiamento:

Nada a declarar.

\section{REFERÊNCIAS}

Almeida, A.M., Godinho, T.M., Bitencourt, A.G., Teles, M.S., Silva, A.S., Fonseca, D.C., ... Oliveira, I.R. (2007). Common mental disorders among medical students. Jornal Brasileiro de Psi- quiatria, 56(4), 245-251. doi: 10.1590/S004720852007000400002

Alves, V.V., Borges, K.C.S., Ribeiro, L.F.P., Gadelha, R.S., \& Santos, S.C. (2010). Concordância entre critérios de categorização do nível de atividade física a partir do questionário internacional de atividade física. Revista Brasileira de Atividade Física e Saúde, 15(2),111-114.

Amaral, G.F., Gomide, L.M.P., Batista, M.P., Píccolo, P.P., Teles, T.B.G., Oliveira, P.M., \& Pereira, M.A.D. (2008). Sintomas depressivos em acadêmicos de medicina da Universidade Federal de Goiás: Um estudo de prevalência. Revista de Psiquiatria do Rio Grande do Sul, 30(2), 124-130. doi: 10.1590/S0101-81082008000300008

Araújo, T.M., Pinho, O.S., \& Almeida, M.M.G. (2005). Prevalência de transtornos mentais comuns em mulheres e sua relação com as características sócio-demográficas e o trabalho doméstico. Revista Brasileira de Saúde Materno Infantil, 5(3), 337-348. doi: 10.1590/S151938292005000300010

Associação Brasileira de Empresas de Pesquisa (ABEP) (2003). Critério de Classificação Econômica Brasil. http://www.marketanalysis.com.br/ar quivos-download/biblioteca/cceb-1.pdf (Acesso em 10 de agosto de 2012).

Baldisserotto, C.M., Filho, E.S., Nedel, F., \& Sakae, T.M. (2005). Problemas psiquiátricos menores e indicadores do uso problemático do álcool entre os estudantes de medicina da Universidade do Sul de Santa Catarina-UNISUL. Arquivo Catarinense de Medicina, 34(4), 73-78.

Ballone, G.J. (2008). O que são transtornos mentais. - In Psiqweb. http://www.psiqweb.med.br (Acesso em 11 de agosto de 2012).

Bray, S.R., \& Born, H.A. (2004). Transition to university and vigorous physical activity: Implications for health and psychological well-being. The Journal of American College Health, 52 (4), 181188. doi: 10.3200/JACH.52.4.181-188

Calais, S.L, Carrara, K., Brum, M.M., Batista, K., Yamada, J.K., \& Oliveira, J.R. (2007). Stresse entre calouros e veteranos de jornalismo. Revista Estudos de Psicologia, 24(1), 69-77. doi: 10.1590/S0103-166X2007000100008

Calfas, K.J., \& Taylor, W.C. (1994). Effects of physical activity on psychological variables in adolescents. Pediatric Exercise Science, 6, 406-423.

Cavestro, J.M., \& Rocha, F.L. (2006). Prevalência de depressão entre estudantes universitários. Jornal Brasileiro de Psiquiatria, 55(4), 264-267. 
Cid, L., Silva, C., \& Alves, J. (2007). Atividade física e bem-estar psicológico: Perfil dos participantes no programa de exercício e saúde de Rio Maior. Motricidade, 3(2), 47-55. doi: 10.6063/motri cidade.3(2).674

Demont-Heinrich, C. (2009). The associations between physical activity, mental health and quality of life: A population-based study. Colorado Health Watch, 71, 1-4.

Facundes, V.L.D., \& Ludemir, A.B. (2005). Common mental disorders among health care students. Revista Brasileira de Psiquiatria, 27(3), 194200. doi: 10.1590/S1516-44462005000300007

Faulkner, G., \& Taylor, A.H. (2005). Exercise health and mental health: Emerging relationships. London, UK: Routledge.

Fiorotti, K.P., Rossoni, R.R., Borges, L.H., \& Miranda, A.E. (2010). Transtornos mentais comuns entre os estudantes do curso de medicina: Prevalência e fatores associados. Jornal Brasileiro de Psiquiatria, 59(1), 19-23.

Fontes, A.C.D., \& Vianna, R.P.T. (2009). Prevalência e fatores associados ao baixo nível de atividade física entre estudantes universitários de uma universidade pública da região Nordeste Brasil. Revista Brasileira de Epidemiologia, 12(1), 20-29. doi: 10.1590/S1415-790X20090001000 03

Guedes, D.P., Santos, C.A., \& Lopes, C.C. (2006). Estágios de mudança de comportamento e prática habitual de atividade física em universitários. Revista Brasileira de Cineantropometria $e$ Desempenho Humano, 8(4), 5-15.

Hallal, P.C., Matsudo, S.M., Matsudo, V.K.R., Araújo, T.M., Andrade, D.R., \& Bertoldi, A.D. (2005). Physical activity in adults from two Brazilian areas: Similarities and differences. Cadernos de Saúde Pública, 21 (2), 573-580. doi: 10.1590/S0102-311X2005000200024

Lawlor, D.A., \& Hopker, S.W. (2001). The effectiveness of exercise as an intervention in the management of depression: Systematic review and meta-regression analysis of randomized controlled trials. British Medical Journal, 322, 763-767. doi: 10.1136/bmj.322.7289.763

Lima, M.C.P., Domingues, M.S., \& Cerqueira, A.T. (2006). Prevalência e fatores de risco para transtornos mentais comuns entre estudantes de medicina. Revista de Saúde Pública, 40(6), 1035-1041. doi: 10.1590/S0034-891020060007 00011
Lino, M.A. (1997). Saúde Mental e Doença Mental Classificação das Doenças Mentais (CID-10) In M.B. Teixeira, I.M. Mello, L.H. Grando, \& D.P. Fraiman (Eds.), Psiquiatria - Manual de Enfermagem. São Paulo: Atheneu.

Ludemir, A.B., \& Filho, D.A.M. (2002). Condições de vida e estrutura ocupacional associados a transtornos mentais comuns. Revista de Saúde Pública, 36(2), 213-121. doi: 10.1590/S003489102002000200014

Marcodelli, P., Costa, T., \& Schmitz, B.A. (2008). Nível de atividade física e hábitos alimentares de Universitários do $3^{\circ}$ ao $5^{\circ}$ semestre da área de saúde. Revista de Nutrição, 21(1), 39-47. doi: 10.1590/S1415-52732008000100005

Mari, J.J., \& Williams, P. (1986). A validity study of a psychiatric screening questionnaire (SRQ-20) in primary care in the city of São Paulo. The British Journal of Psychiatry, 148, 23-26. doi: 10.1192/bjp.148.1.23

Matsudo, S., Araújo, T., Matsudo, V., Andrade, D., Andrade, E., Oliveira, L., \& Braggion, G. (2001). Questionário Internacional de Atividade Física (IPAQ): Estudo de validade e reprodutibilidade no Brasil. Revista Brasileira de Atividade Física \& Saúde, 6(2), 5-18.

Nahas, M.V., \& Fonseca, S.A. (2004). Estilo de vida e hábitos de lazer dos trabalhadores da indústria catarinense (1999-2004). Florianópolis: SESI.

Polydoro, S.A.J., Primi, R., Serpa, M.N.F., Zaroni, M.M.H., \& Pombal, K.C.P. (2001). Desenvolvimento de uma escala de integração ao ensino superior. Psico-USF, 6(1), 11-17. doi: 10.1590/ S1413-82712001000100003

Rodrigues, E.S., Cheik, N.C., \& Mayer, A.F. (2008). Nível de atividade física e tabagismo em universitários. Revista de Saúde Pública, 42(4), 672678. doi: 10.1590/S0034-89102008000400013

Roeder, M.A. (1999). Benefícios da atividade física em pessoas com transtorno mental. Revista Brasileira de Atividade Física e Saúde, 4(2), 62-76.

Sakae, T.M., Padão, D.L., \& Jordana, L.K. (2010). Sintomas depressivos em estudantes da área da saúde em uma universidade no sul de Santa Catarina - UNISUL. Revista da Associação Médica do Rio Grande do Sul, 54(1), 38-43.

Scully, D., Kremer, J., Meade, M.M., Grahan, R., \& Dudgeon, K. (1998). Exercise and psychological well being: A critical review. British Journal of Sports Medicine, 32, 111-120. doi: 10.1136/bjsm. 32.2.111 
Silva, G.S.F., Bergamaschine, R., Rosa, M., Melo, C., Miranda, R., \& Bara Filho, M. (2007). Avaliação do nível de atividade física de estudantes de graduação da área de saúde/biológica. Revista Brasileira de Medicina do Esporte, 13(1), 32-35. doi: 10.1590/S1517-86922007000100009

Silva, R.S., Silva, I., Silova, R.A., Souza, L., \& Tomazi, E. (2010). Atividade física e qualidade de vida. Revista Ciência \& Saúde Coletiva, 15(1), 115120. doi: 10.1590/S1413-81232010000100017
Souza, T.F., Santos, S.F.S., \& José, H.P.M. (2010). Práticas de atividade física no lazer e barreiras percebidas dessa prática em acadêmicos de um curso de educação física no nordeste do Brasil. Revista Pensar a Prática, 13(1), 1-15.

Tondo, J., Silva, T., \& Roth, M. (2011). Barreiras percebidas e nível de atividade física de universitários residentes na casa do estudante da Universidade Federal do Sul do Brasil. Lecturas: Efdeportes.Com, 15(153).

Todo o conteúdo da revista Motricidade está licenciado sob a Creative Commons, exceto quando especificado em contrário e nos conteúdos retirados de outras fontes bibliográficas. 